\title{
Quali ragioni e quali possibili soluzioni per il mancato sviluppo della dialisi peritoneale in Italia?
}

\author{
Amedeo F. De Vecchi \\ Divisione di Nefrologia e Dialisi \\ IRCCS Ospedale Maggiore Policlinico, Milano
}

na ormai lunga esperienza in dialisi peritoneale mi ha convinto che questo tipo di trattamento ha enormi potenzialità; in molte situazioni consente una buona sopravvivenza e una qualità di vita addirittura migliore dell'emodialisi, soprattutto nei pazienti giovani e/o in quelli idonei al trapianto.

Credo che diversi fattori concorrano e abbiano concorso al mancato sviluppo della dialisi peritoneale. Cercherò di riassumere in queste pagine le mie impressioni, spesso non condivise, sostenendole ove possibile con un' adeguata bibliografia.

Innanzi tutto molti nefrologi sono stati educati quando ancora la dialisi peritoneale cronica era gravata da troppi rischi di infezioni e ritengono che questa metodica possa essere utile solo per trattare i pazienti non idonei alla emodialisi o con scarsa possibilità di riabilitazione. Con queste idee sono stati indirizzati alla dialisi peritoneale i pazienti con breve attesa di vita, e/o con numerosi fattori di rischio. Ovviamente in tali situazioni il rischio d'insuccessi, complicanze (anche non correlate alla dialisi), ricoveri e mortalità è estremamente elevato e ha portato molti ad approfondire la propria diffidenza per questa metodica. Negli ultimi anni, malgrado tutta la letteratura dimostri il contrario (1-3), molti nefrologi o chirurghi continua- no a ritenere la dialisi peritoneale una controindicazione al trapianto. Inoltre la dialisi peritoneale richiede un'attenzione e una "condotta terapeutica" un po' diverse, se non maggiori, rispetto all'emodialisi. Infatti la dialisi peritoneale consente, ma anche spesso richiede, una individualizzazione del protocollo di trattamento, teso soprattutto al mantenimento della diuresi residua e della qualità di vita. La dialisi peritoneale richiede la presenza di un catetere, con i relativi rischi infettivi, e utilizza una membrana biologica, con tutti i rischi di invecchiamento e danno e loro conseguenze. Infine la dialisi domiciliare e autogestita impone una più assidua e disponibile assistenza al paziente. Pertanto i protocolli rigidi sono spesso dannosi, il mantenimento della diuresi residua va perseguito anche a costo di mantenere il paziente moderatamente iperidratato $\mathrm{e}$ così via. Oserei affermare che la dialisi peritoneale va quasi sempre ritagliata su misura per il singolo paziente, come orari, volumi, tipo e numero di scambi impedendo una gestione superficiale o massificata.

Ovviamente non dobbiamo dimenticare che la dialisi peritoneale utilizza una membrana (il peritoneo) estremamente biocompatibile, ma a bassa efficienza e non sostituibile quando venga danneggiata. Pertanto una cor- retta condotta implica che, quando il peritoneo non sia più in grado di depurare o ultrafiltrare, il paziente sia rapidamente passato all'emodialisi (e non all'APD) per evitare inutili 'trascinamenti' con conseguenti complicazioni e ricoveri.

Nel questionario sottoposto a Taormina, che purtroppo pochi hanno compilato, tra le indicazioni alla dialisi peritoneale la volontà del paziente ha ricevuto scarsa attenzione, anche se costituisce il cardine di qualunque trattamento domiciliare. I partner, per quanto affettuosi ed altruisti, non possono sostituire il paziente al $100 \%$ e la grave limitazione delle loro possibilità di vita pesa enormemente sulla qualità del loro aiuto.

Tra le ragioni non mediche del mancato sviluppo della dialisi peritoneale, ritengo che i rimborsi abbiano un'importanza relativa; la dialisi peritoneale ha un rimborso vantaggioso solo se sono trattati un certo numero di pazienti, ma costituisce un pool mobile per l'emodialisi, permettendo di gestire un numero maggiore di pazienti, il che in un regime di budget $\mathrm{e}$ incentivi non è certo un vantaggio da poco... A proposito di rimborsi vorrei sottolineare che le tariffe per la dialisi peritoneale sono ormai vicine a quelle dell'emodialisi standard, pur essendo i costi unanimemente considerati inferiori almeno del 20\% (4-6). 
Un secondo aspetto che potrebbe nuocere alla dialisi peritoneale, è il mancato utilizzo di questa metodica nei Centri privati, che porta soprattutto questi ultimi a propagandare in modo più o meno corretto, l'emodialisi come migliore trattamento.

Anche a costo di tirarmi addosso grossi dissensi, ritengo che anche gli investimenti e la propaganda dell'industria verso la dialisi peritoneale automatizzata abbiano danneggiato la dialisi peritoneale. Infatti, affermando che la dialisi automatizzata offre una migliore efficienza (7) (ma parliamo solo di numeri!!) e una migliore qualità di vita (8) (mai dimostrata in modo incontrovertibile), molti hanno appreso più o meno chiaramente che la dialisi peritoneale non è adeguata, che gli scambi nuocciono alla qualità di vita del paziente e così via. Sono convinto che qualunque tipo di dialisi riduca la qualità di vita, ma non credo che la maggior parte dei pazienti ritengano l'APD meno traumatizzante della CAPD, come non credo che preferiscano l'emodialisi alla CAPD (10). Questa campagna ha portato anche a un'esasperazione dell'importanza del $\mathrm{Kt} / \mathrm{V}$ e a una gestione meccanicistica e numerocentrica della dialisi peritoneale. L'adeguatezza è secondo me un concetto clinico complesso che non può e non deve mai essere rappresentato da un singolo numero (11): i numeri disponibili sono stati validati solo parzialmente $e$ in modo indiretto in alcuni lavori non prospettici né controllati (12-14). Per perseguire il numero si dimentica la clinica, il paziente e la finalità del trattamento. Un nostro paziente, trattato con dialisi peritoneale per 18 anni, ha avuto sempre una situazione clinica di dialisi adeguata, anche se i Kt/V spesso erano sotto i valori canonici di 1.8-2.

Mentre molti pazienti passano dalla CAPD alla APD per questi presunti e non dimostrati vantaggi, è sorprendente che quasi nessuno, trovandosi male in APD, passi alla metodica manuale, ma venga piuttosto trasferito in emodialisi. Queste mie tesi non sono avallate da alcuno studio scientificamente incontrovertibile, ma è evidente, almeno in Italia, un diffuso declino della dialisi peritoneale che coincide (casualmente?) con la promozione e l'espansione dell'APD, dopo un decennio di costante progresso della CAPD. In realtà la dialisi peritoneale automatizzata presenta notevoli vantaggi gestionali per alcuni pazienti, ma per altri è un'importante fonte d'ansia, d'insonnia, d'ulteriori problemi. Pertanto, come sempre in medicina, CAPD ed APD sono metodiche complementari, non antitetiche, come alcuni vogliono far credere e il loro utilizzo deve essere dettato dal buon senso clinico e non dagli umori e dalle mode.

Quali sono in sintesi i vantaggi della dialisi peritoneale:

Clinici: migliore riabilitazione (assenza di picchi), maggiore libertà di movimento e vacanza, gestione domiciliare, mantenimento della diuresi residua, migliore controllo dell'anemia, della pressione, della fosfatemia.

Organizzativi: consente di dializzare numerosi pazienti con minimi investimenti di spazi e di personale, e quindi aumenta il pool di pazienti in trattamento sostitutivo, consentendo, se ben utilizzata, attivi maggiori.

Gli svantaggi invece sono così riassumibili:

Clinici: minore durata dell'efficienza dialitica (rispetto all'HD), maggiore rischio di malnutrizione (?), complicanze infettive.

Organizzativi: necessità di personale almeno parzialmente dedicato, ambulatorio, preparazione di protocolli, più frequenti cambi di terapia, maggiori ricoveri (?).

A scopo provocatorio, per stimolare discussione e quindi approfondimento del problema, suggerirei alcuni punti per migliorare l'utilizzo e lo sviluppo della dialisi peritoneale.

\section{Clinica}

1) Utilizzare la dialisi peritoneale nei pazienti con corrette indicazioni e soprattutto con possibilità e volontà di eseguire un trattamento domiciliare (i pazienti sen- za queste caratteristiche, in cui la dialisi peritoneale è l'ultima spiaggia, non devono essere considerati per i risultati del trattamento);

2) cercare di mantenere la diuresi residua, anche a costo di una modesta iperidratazione;

3) basare la quantità di dialisi tenendo conto prima della situazione clinica e poi anche dei "numeri";

4) essere estremamente elastici nella prescrizione degli orari e discutere con il paziente eventuali scambi aggiuntivi o passaggi ad altre metodiche;

5) considerare sempre il paziente e non la terapia, cercando di utilizzare tutte le terapie disponibili, anche in sequenza rapida, per perseguire la maggiore riabilitazione del paziente: non è un insuccesso passare un paziente da una terapia all'altra, ma è un insuccesso non riabilitarlo e non soddisfare le sue esigenze;

6) iniziare la dialisi tempestivamente.

\section{Organizzazione}

1) Proporre tempestivamente al paziente tutte le opzioni dialitiche, in modo imparziale, e far scegliere a lui, in assenza di gravi controindicazioni cliniche, il tipo di dialisi più adatto;

2) non "promuovere" mai un trattamento rispetto a un altro;

3) eventualmente includere nei contratti d'acquisto dei materiali per dialisi peritoneale anche parte dell'addestramento (in ospedale o a domicilio) da parte delle infermiere delle ditte fornitrici;

4) in situazioni di scarsa esperienza o di numeri di pazienti troppo esigui consorziarsi con ospedali vicini o con Centri più grossi con convenzioni o altro, soprattutto per le metodiche chirurgiche;

5) cercare di iniziare la dialisi peritoneale con pazienti idonei, non con scarti;

6) se possibile utilizzare medici e infermieri dedicati e 'volontari', evitando che la dialisi peritoneale diventi il trattamento seguito dai medici che non hanno mai trovato 
un loro ruolo o da infermieri invalidi o inadatti ad altri compiti;

7) approfondire la cultura dialitica estendendola alla dialisi peritoneale.

\section{Politica sanitaria}

1) Premiare i Centri che abbiano una percentuale di pazienti in dialisi peritoneale superiore ad un certo numero;

2) incentivare l'utilizzo delle dialisi meno costose (rimborso pro capite per i dializzati?, da incrementarsi eventualmente per ogni anno di trattamento?);

3) consentire convenzioni per permettere, con adeguati controlli qualitativi, l'utilizzo della dialisi peritoneale in Centri privati;

4) imporre (e consentire) ai Centri privati una convenzione con Centri esperti per la collaborazione ed il controllo di qualità della dialisi peritoneale;

5) programmare convenzioni con ospedali o società di servizi, per accentrare parzialmente il followup dei pazienti seguiti da Centri con scarsa esperienza e/o minima casistica;

6) impedire che l'arrivo di un nuovo dirigente di struttura complessa, che non creda nella peritoneale, ne riduca o annulli l'incidenza.

deveccpd@polic.cilea.it

\section{BIBLIOGRAFIA}

1. Péréz Fontàn M, RodríguezCarmona A, Garcìa Falcón T, et al. Peritoneal dialysis is not a risk factor for primary vascular graft thrombosis after renal transplantation. Perit Dial Int 1998; 18 (3): 311-6.

2. Lambert MC, Bernard P, Vijt $D$, et al. CAPD a risk factor in renal transplantation? ARF after transplantation. Perit Dial Int 1996; 16 (suppl.): S495-8.

4. Ruggieri G, Brunori G, De Vecchi A et al. Il costo sociale della dialisi. N Di Paolo, U Buoncristiani eds. In: Tecniche nefrologiche e dialitiche. Milano, Wichtig, 1994; 405-15.

5. Piccoli G, Formica M, Mangiarotti $\mathrm{G}$, et al. The costs of dialysis in Italy. Nephrol Dial Trans 1997; 12 (suppl.) S33-44.

6. De Vecchi AF, Dratwa M, Wiedermann M. Healthcare systems and ESRD therapies - An international review. Costs and reimbursement/ funding of ESRD therapies. Nephrol Dial Transplant 1999; 14 (suppl.): S31-41.

7. Misra S, Nolph KD, Khanna R. Will automated peritoneal dialysis be the answer? Perit Dial Int 1997; 17 (5): 435-9.

8. Blake PG. Targets in CAPD and APD pescription. Perit Dial Int 1996; 16 (suppl.): S143-6.

9.

Holley JL, Piraino B. Careful patient selection and dialysis prescription are required for effective nightly intermittent peritoneal dialysis. Perit Dial Int 1994; 14 (2): 155-8.

10. Wrangler E, Krautzig S, Bronkhorst R. Adequacy and quality of life with automated peritoneal dialysis, Perit Dial Int 1996; 16 (suppl.): S153-7.

11. De Vecchi A, Scalamogna A, Colombini M, et al. Well being in patients on CAPD and hemodialysis. Int J Artif Organs 1994; 17: 473-7.
12. Ronco C. Adequacy of peritoneal dialysis is more than Kt/V. Nephrol Dial Transplant 1997; 12 (suppl.): S68-73.

13. Adequacy of dialysis and nutrition in continuous peritoneal dialysis: association with clinical outcomes. Canada USA (CANUSA). Peritoneal Dialysis Study Group. J Am Soc Nephrol 1996; 7 (2): 198-207.

14. Blake P. A critique of the Canadian USA (CANUSA) peritoneal dialysis study. Perit Dial Int 1996; 16: 243-5.

15. Brandes JC, Piering WF, Beres JA et al. Clinical outcome of continuous peritoneal dialysis predicted by urea and creatinine kinetics. J Am Soc Nephrol 1992; 2: 1430-5.

16. De Alvaro F, Bajo MA, Alvarez Ude et al. Adequacy of peritoneal dialysis: does KT/V have the same predictive value as in HD? a multicenter study. Adv Perit Dial 1992; 8: 93-7.

17. Keshaviah P. Adequacy of CAPD a quantitative approach. Kidney Int 1992; 42: 160-4.

18. Keshaviah P. Pitfalls in measuring peritoneal dialysis prescription Periton Dial Int 1994; 14 (suppl.): S88-92. 